\title{
Quantized Orbits and Resonant Transport
}

\author{
Indubala I. Satija* \\ Department of Physics, George Mason University, Fairfax, VA 22030 \\ Bala Sundaram ${ }^{\dagger}$ \\ Department of Mathematics, CSI-CUNY, 2800, Victory Boulevard Staten Island, NY 10314
}

(July 15, 2018)

\begin{abstract}
A tight binding representation of the kicked Harper model is used to obtain an integrable semiclassical Hamiltonian consisting of degenerate "quantized" orbits. New orbits appear when renormalized Harper parameters cross integer multiples of $\pi / 2$. Commensurability relations between the orbit frequencies are shown to correlate with the emergence of accelerator modes in the classical phase space of the original kicked problem. The signature of this resonant transport is seen in both classical and quantum behavior. An important feature of our analysis is the emergence of a natural scaling relating classical and quantum couplings which is necessary for establishing correspondence.

03.65.Sq,05.45.Mt,64.60.Ak
\end{abstract}

Typeset using REVTEX

*email:isatija@sitar.gmu.edu

†email:bas@math.csi.cuny.edu 
The search for evidence of complexity in quantum systems when the corresponding classical system exhibits chaotic dynamics is an active frontier in nonlinear science. Kicked systems with classically chaotic dynamics have provided useful theoretical [1].2] and experimental [3] paradigms for elucidating the fundamental issues of quantum chaos. Systems where quantum states can be either extended or localized provide the added challenge of finding signatures of the localization-delocalization transition in the corresponding classical system. In this letter, we consider the kicked Harper system to establish precisely such a correspondence, within the context of fascinating issues such as anomalous transport [4] and dynamical localization [2].

The traditional approach starts with the classical analysis and then searches for signatures in the quantum behavior. By contrast, we begin with the quantum model and, using a novel semiclassical approach, predict a series of enhancements to transport in the system. These 'phase transitions' are shown to correspond to ballistic to diffusive transitions in both classical and quantum dynamics, and are due to classical accelerator modes. These modes, in turn, are known to result in anomalous transport 团. Our methodology is applicable to a variety of kicked systems. However, we will illustrate our method in the kicked Harper model,

$$
H=L \cos (p)+K \cos (q) \sum_{k} \delta(t-k),
$$

where the relative values of the parameters $K$ and $L$ determine the dynamics. The classical dynamics reduces to the two-dimensional area preserving Harper map,

$$
\begin{aligned}
& p_{k+1}=p_{k}+K \sin \left(q_{k}\right) \\
& q_{k+1}=q_{k}-L \sin \left(p_{k+1}\right) .
\end{aligned}
$$

This mapping is analogous to the standard map or kicked rotor, with the dynamics becoming globally chaotic when both $K$ and $L$ cross critical values. However, neither system is ergodic as islands, arising via bifurcations, exist in the chaotic sea leading to the possibility of anomalous transport. In particular, hierarchical island chains for $K=L$ in the vicinity of $2 \pi$ have been shown to result in fractional kinetics [4.

By contrast, the quantum dynamics of the kicked Harper model is quite different from that of the quantum kicked rotor. Most significant is the existence of both extended and localized states depending on $K$ and $L$. Earlier studies indicate that extended states exist for $K>L$ while localization occurs for $K<L$ [5], even though the $K=L$ critical line, demarcating the onset of localization, has been rigorously established only in the absence of kicking. The existence of this transition allows the possibility of both ballistic as well as diffusive transport in the system.

Our analysis emphasizes the dynamics along the line $K=L$ and, using a novel semiclassical approach, predicts a rich variety of behavior. The key is the mapping of the temporal dynamics of the quantum kicked model to a continuum time-independent integrable Hamiltonian, within which the complexity of the original problem manifests itself in terms of degenerate classical orbits. These orbits are indexed by an integer $n$, where the $n^{\text {th }}$ orbit appears when the renormalized coupling $\bar{K}=K /(2 \hbar)=\bar{L}$ exceeds $n \pi / 2$. The phase space of this semiclassical Hamiltonian resembles the unkicked Harper model except that only certain orbits are allowed. Therefore, in this semiclassical formulation, kicking the system selects a 
discrete number of classical trajectories of the unkicked Harper system. These additional orbits are shown to enhance both classical and quantum transport in the kicked Harper model. Finally, classical and quantum results display good correspondence provided the classical parameter is set to be $\bar{K}$. This implies an $\hbar$ scaling considerably different from that used earlier [6].

We start from the association of the time-dependent quantum Hamiltonian with a tightbinding lattice problem [2]. This is obtained by projecting individual quasienergy states, associated with the single-kick evolution operator, onto angular momentum eigenstates $\mid m>$ with eigenvalues $p=m \hbar$. The projection coefficients $u_{m}$ satisfy

$$
\sum_{r} J_{r}(\bar{K}) \sin \left[\frac{1}{2}(\omega-2 \bar{L} \cos (m \hbar))-\frac{\pi r}{2}\right] u_{m+r}=0,
$$

where $\omega$ is the quasienergy corresponding to the eigenstate. Following Wilkinson [7], we consider the continuum limit of the lattice model by defining $x_{m}=m \hbar$ and $\psi\left(x_{m}\right)=\psi_{m}$. Thus, $\left(\psi_{m+r}+\psi_{m-r}\right)$ is replaced by $2 \cos (r \pi p) \psi\left(x_{m}\right)$ as $p$ is the generator of space translation. Taking $\omega=0$, the tight-binding model can be written as $H \psi(x)=0$, where the Hamiltonian operator $H$ is,

$$
\begin{aligned}
& H=\sin (\bar{L} \cos (x))\left[J_{0}(\bar{K})+2 \sum(-1)^{r} J_{2 r}(\bar{K}) \cos (2 r p)\right] \\
& +\cos (\bar{L} \cos (x))\left[2 \sum(-1)^{r-1} J_{2 r-1}(\bar{K}) \cos ((2 r-1) p)\right] .
\end{aligned}
$$

Note that the lattice problem $H \psi(x)=0$ can be viewed as an eigenvalue problem restricted to a Hilbert subspace defined by eigenvalue $E=0$. The terms in the square brackets $H$ can be summed exactly to give $\cos (\bar{K} \cos (p))$ and $\sin (\bar{K} \cos (p))$ respectively. Therefore, the resulting semiclassical Hamiltonian simplifies to

$$
H_{s c l}=\sin [\bar{K} \cos (p)+\bar{L} \cos (x)] .
$$

The fact that the Hamiltonian dynamics is constrained to $E=0$ implies that the classical phase space trajectories satisfy

$$
\bar{K} \cos (p)+\bar{L} \cos (x)= \pm n \pi,
$$

where $n$ is an integer. This can be viewed as the "quantization condition" for the phase space of the unkicked Harper equation given by $\bar{K} \cos (p)+\bar{L} \cos (x)=E$. Thus, within this semiclassical formulation, the kicking effectively quantizes the classical orbits. Another interesting feature, which we exploit later, is the fact that $\hbar$ appears only in $\bar{K}$ and $\bar{L}$ and not as an independent parameter. Changing $\hbar$ simply renormalizes the couplings $K$ and $L$. Note also that $H_{s c l}$ is symmetric in $x$ and $p$.

The phase portrait resulting from $H_{s c l}$ consists of degenerate orbits indexed by the integer $n$. For $\bar{K}<\pi / 2$ and $\bar{L}<\pi / 2$, there exists only a single orbit and additional degenerate branches appear as $\bar{K}$ and $\bar{L}$ exceed threshold values $\bar{K}_{n}^{*}=n \pi / 2$. The quantized orbits are not necessarily closed, unless $\bar{K}=\bar{L}$. For the rest of the discussion, we will confine ourselves to this symmetric case. In the corresponding quantum problem, the condition $\bar{K}=\bar{L}$ for closed orbits suggests a phase boundary for a localization-delocalization transition [7]. For $\bar{K}<\pi / 2$ this boundary is the separatrix labeled by $n=0$. Therefore, $\bar{K}=\pi / 2$, when the 
next orbit appears, can be viewed as the semiclassical threshold for the onset of diffusion in the kicked Harper rotor [8].

The frequencies of the degenerate orbits are obtained from the equations of motion resulting from $H_{s c l}$

$$
\begin{aligned}
& \dot{p}=\bar{K}(-1)^{n} \sin (x) \\
& \dot{x}=-\bar{K}(-1)^{n} \sin (p) .
\end{aligned}
$$

For any $\bar{K}$, the frequency of the $n^{\text {th }}$ orbit is given, in terms of the complete elliptic function $\mathcal{K}$, by

$$
f_{n}=\bar{K} /\left[4 \mathcal{K}\left(\alpha_{n}\right)\right]
$$

where the modulus $\alpha_{n}=\sqrt{\left[1-\left(\bar{K}_{n}^{*} / \bar{K}\right)^{2}\right]}$. The turning points of these quantized orbits (for $n>0) x=2 \cos ^{-1}\left(\sqrt{\bar{K}_{n}^{*} / \bar{K}}\right)$ imply that, at $\bar{K}=\bar{K}_{n}^{*}$, the new orbits appear at the origin (similarly, for $n<0$ they occur at $(\pi, \pi)$ ). At threshold, the $n^{\text {th }}$ orbit frequency $f_{n}^{*}=n / 4$ is commensurate with the kicking frequency which was taken to be unity (see (1) ). As $\bar{K}$ increases, the frequencies increase monotonically and are, in general, incommensurate except at special parameter values. For example, for $\bar{K} \approx 1.4 \pi$, we have $f_{1} / f_{2}=4 / 3$. $f_{1}$ and $f_{2}$ are commensurate with the kicking frequency at several values: $f_{1}=1 / 3$ at $\bar{K} \approx .86 \pi$, and $f_{1}=1 / 2$ at $\bar{K} \approx 1.68 \pi$ while $f_{2}=2 / 3$ at $\bar{K} \approx 1.71 \pi$. The significance of these 'critical' $\bar{K}$ values will be seen shortly.

In general, the quantum transport is expected to be enhanced by tunneling between these classically distinct orbits [7]. Whenever a threshold value $\bar{K}_{n}^{*}$ is crossed, a new branch appears which may enhance the tunneling probability. We further expect that the transport may also be influenced by the commensurability relations between the competing orbital and kicking frequencies.

Motivated by the possibility of phase transitions leading to the enhancement of diffusion, we now investigate classical as well as quantum transport in the original kicked Harper model. Some of the threshold values predicted by our semiclassical analysis are curiously reminiscent of known special values $K=L=2 \pi m$ beyond which accelerator modes appear in the Harper map. These are known to result in either ballistic or anomalous transport. On identifying $\bar{K}$ with the classical kicking parameter, the $\bar{K}_{n}^{*}$ values include the thresholds for accelerator modes. This scaling emerges naturally from our semiclassical analysis and motivates the investigation of classical transport in the neighborhood of $K=\bar{K}_{n}^{*}=n \pi / 2$.

Interestingly, a number of secondary fixed points of the classical map (eq.(2)) appear at $\bar{K}_{n}^{*}$. Of these, a large number are accelerator modes, including the low-period ones associated with fractional kinetics [四]. For example, at $\bar{K}=2 n \pi$, four period-1 fixed points at $( \pm \pi / 2, \pm \pi / 2)$ appear on $p=q$ and $p=-q$ symmetry lines. Increasing $\bar{K}$ leads to pairs of period-1 points straddling the symmetry lines and, finally, hierarchies of island chains which result in anomalous transport. The parametric window for this behavior extends till $\bar{K} \approx 6.6$. For odd-multiples of $\pi(\bar{K}=(2 n+1) \pi)$, analogous behavior with period-2 accelerator modes, one of which is $(0, \pi / 2) \rightarrow(\pi / 2,0) \rightarrow\left(0, \pi / 2+2 \pi l_{K}\right)$, is seen. The island hierarchies at larger $K$ are exemplified in Figure. 1 1 (b). Topological structures consisting of boundary layer island chains and, closer to the thresholds, the more exotic blinking and shrinking/blowing islands [4.9] strongly suggest anomalous transport within parametric windows. Additional 
accelerator modes also appear at certain critical values, in particular, we have period-2 modes at $\bar{K} \approx .87 \pi$ and $\bar{K} \approx 1.38 \pi$ on the $p=q$ symmetry line. Note that these are close to parameters for the commensurability conditions $f_{1}=1 / 3$ and $f_{1} / f_{2}=4 / 3$, respectively.

The classical behavior at $\bar{K}=(n+1 / 2) \pi$ is more interesting with period-6 fixed points appearing on the symmetry lines $p=0, q=0, p=q$, and $p=-q$. As illustrated by the iterates $(\pi / 2, \pi / 2) \rightarrow(\pi / 2,(n+1) \pi) \rightarrow((n+1) \pi,(2 n+3 / 2) \pi) \rightarrow((2 n+3 / 2) \pi,(2 n+$ $3 / 2) \pi) \rightarrow((2 n+3 / 2) \pi,(n+1) \pi) \rightarrow((n+1) \pi, \pi / 2) \rightarrow(\pi / 2, \pi / 2)$, these fixed points are non-accelerating despite leaving the fundamental interval $[0,2 \pi]$ at intermediate steps. As $\bar{K}$ increases, these orbits also move off the symmetry lines as stable islands, as seen from Fig. 1(a). Even though the fixed point is non-accelerating, an initial condition in the vicinity of the fixed point returns outside the fundamental domain after 6 iterations. In this sense, accelerated transport can occur at these $\bar{K}$.

The effect of these accelerator modes on classical transport is illustrated in Fig. 2(a) in terms of the exponent $\mu$ defined by $\left\langle p^{2}>\propto t^{\mu}\right.$, where the braces denote an ensemble average [10]. Many spikes in $\mu$ are seen as the parameter $\bar{K}$ is changed. The sharp, resonant, structures corresponding to ballistic transport, $\mu \approx 2$, are due to the narrow ranges of stability of the accelerator modes. Interestingly, these peaks appear very close to the $K_{n}^{*}$ values as well as $\bar{K}$ values where the orbital frequencies exhibit commensurability. A few of these special values are indicated by the arrows in the figure. Thus our quantum analysis is providing information about the classical transport properties. Note that for smaller $\bar{K} / \pi \approx 0.6$, a stochastic web dominates the classical transport.

As shown in earlier work [4, 11], enhancement of the quantum localization length $\xi$ can be used as a signature of classical ballistic behavior. Here we utilize this idea while, at the same time, contrasting the scaling suggested by our analysis with that used earlier [4,60.11. The resonant transport in classical and quantum behavior shows good correspondence provided the classical stochasticity parameter is taken to be $\bar{K}=K /(2 \hbar)$. This is quite different from $K_{q}=K \sin (\hbar / 2) /(\hbar / 2)$ obtained earlier [6] by contrasting correlations in the classical and quantum dynamics. $\bar{K}$ emerges naturally from a 'thermodynamic' formalism, as the tight-binding (eigenvalue) problem deals with only a single quasienergy state 12]. As such, this may be valid beyond a certain time scale. We consider moderate values of $\hbar$ and, as seen from panels Fig. 2(b) and(c), spikes in $\xi$ appear close to classical values only when $\bar{K}$, and not $K_{q}$, is considered. At these values of $\hbar$, the issue of reaching large $t$ behavior does not arise. Also, as in earlier work [11], the quantization scale is much larger than the size of the classical structures causing the accelerated transport. The intriguing possibility, for smaller $\hbar$, of a cross-over from $K_{q}$ to $\bar{K}$ at longer times warrants further exploration.

Figure 3 shows the quantum lineshape at one of the peaks (a) contrasted with the shape away from the peak (b). It is clear that the quantum transport at the peak has a ballistic component which results in the well-defined shoulders in Fig. 3(a) as compared with panel (b).

Despite the unphysical nature of the Harper system, resonant transport at $K_{n}^{*}$ and other critical values can be tested experimentally. As the origin of resonances has been related to accelerator modes, analogous behavior exists in the kicked rotor (standard map) [3, 13] and oscillator (web map), both of which are experimentally realizable in atom optics. In fact, even the question of scaling as discussed above could also be resolved experimentally in this context. 
The research of IIS is supported by a grant from National Science Foundation DMR 097535. The work of BS was supported by the National Science Foundation PHY 9800966 and a grant from the City University of New York PSC-CUNY Research Award Program. 


\section{REFERENCES}

[1] Quantum Chaos: between order and disorder: a selection of papers, edited by G. Casati and B. V. Chirikov (Cambridge University Press, New York, 1995).

[2] S. Fishman, D. R. Grempel, and R. E. Prange, Phys. Rev. Lett. 49, 509 (1982); Phys. Rev. A 29, 1639 (1984).

[3] F. L. Moore, J. C. Robinson, C. F. Bharucha, Bala Sundaram, and M. G. Raizen, Phys. Rev. Lett. 75, 4598 (1995); B. G. Klappauf, W. H. Oskay, D. A. Steck, and M. G. Raizen, Phys. Rev. Lett. 81, 4045 (1998). C. F. Bharucha, J. C. Robinson, F. L. Moore, Bala Sundaram, Qian Nu, and M. G. Raizen, Phys. Rev. E 60 (1999).

[4] G. Zaslavsky and B. A. Niyazov, Phys Report 283, 73 (1997); "Immense delocalization from fractional Kinetics', A. Iomin and G. M. Zaslavsky, CHAOS (in press).

[5] R. Lima and D. Shepelyansky, Phys. Rev. Lett. 67, 1377 (1991).

[6] D. L. Shepelyansky, Physica 28D, 103 ( 1987).

[7] M. Wilkinson, Proc. R. Soc. London A391, 305 (1984).

[8] This value is reminiscent of the threshold value for the onset of global diffusion obtained from the resonance overlap criterion.

[9] G. M. Zaslavsky, M. Edelman, and B. A. Niyazov, CHAOS 7, 159 (1997).

[10] $\mu$ simply reflects the existence of ballistic transport over some fraction of the time period considered, which is sufficient for our purposes.

[11] Bala Sundaram and G. M. Zaslavsky, Phys. Rev. E 59, 7231 (1999).

[12] With this view, the singularity as $\hbar \rightarrow 0$ may reflect the well-known non-commutativity of this limit with the $t \rightarrow \infty$ limit.

[13] Many of the semiclassical predictions discussed here are also valid for standard kicked rotor. The thresholds change to integer multiples of $\pi$ where an open trajectory becomes closed, proving a new competing frequency in the semiclassical Hamiltonian. 


\section{FIGURES}

FIG. 1. Phase space portraits of the accelerator islands beyond the thresholds $K=\bar{K}_{n}^{*}=n \pi / 2$. (a) $K=5.04(n=3)$ where one of the six islands is shown; (b) $K=3.199(n=4)$ where only one of four symmetric structures is shown. Note the size of these structures in relation to the $\hbar$ values considered.

FIG. 2. (a)Variation of the classical transport exponent $\mu$ with the classical stochasticity parameter set to $\bar{K}$. An ensemble of 1001 initial conditions along the line $p=0$ was iterated $10^{5}$ times, and the last $5 \times 10^{4}$ iterations were used to obtain $\mu$. The arrows at the top indicate some of the special values arising from commensurability and thresholds $\bar{K}_{n}^{*}$ (see text). (b)-(c) Variation of the localization length $\xi$ with $\bar{K}$ (solid) and $K_{q}$ (dashed). $\hbar=2 \pi \sigma^{M}$ and (b) $M=1$ and (c) $M=3$, where $\sigma$ refers to the golden mean. A fixed interaction time of 4000 kicks was considered.

FIG. 3. Quantum lineshape at the end of 4000 kicks. (a) $\bar{K}=6.4402648$ while (b) $\bar{K}=6.3146012 . \hbar=2 \pi \sigma^{3}$ and case (a) is at a peak in Figure 2(c) while (b) is off the peak. 


\section{FIGURES}
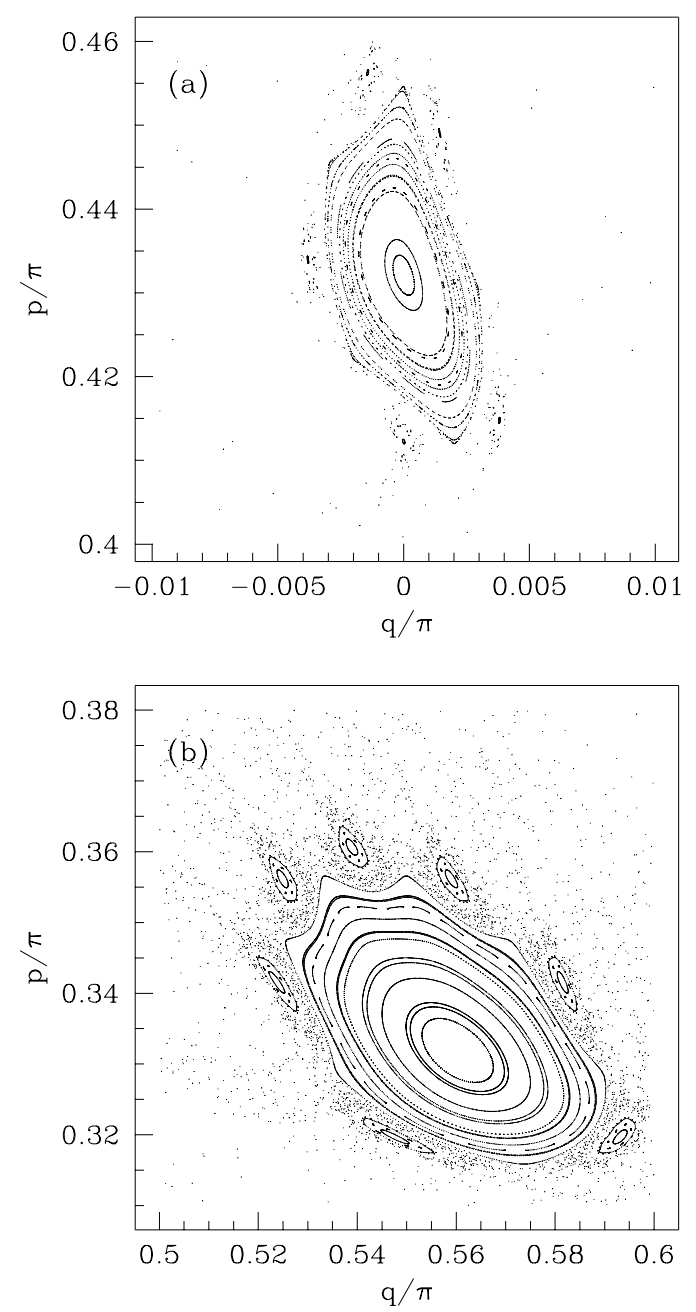

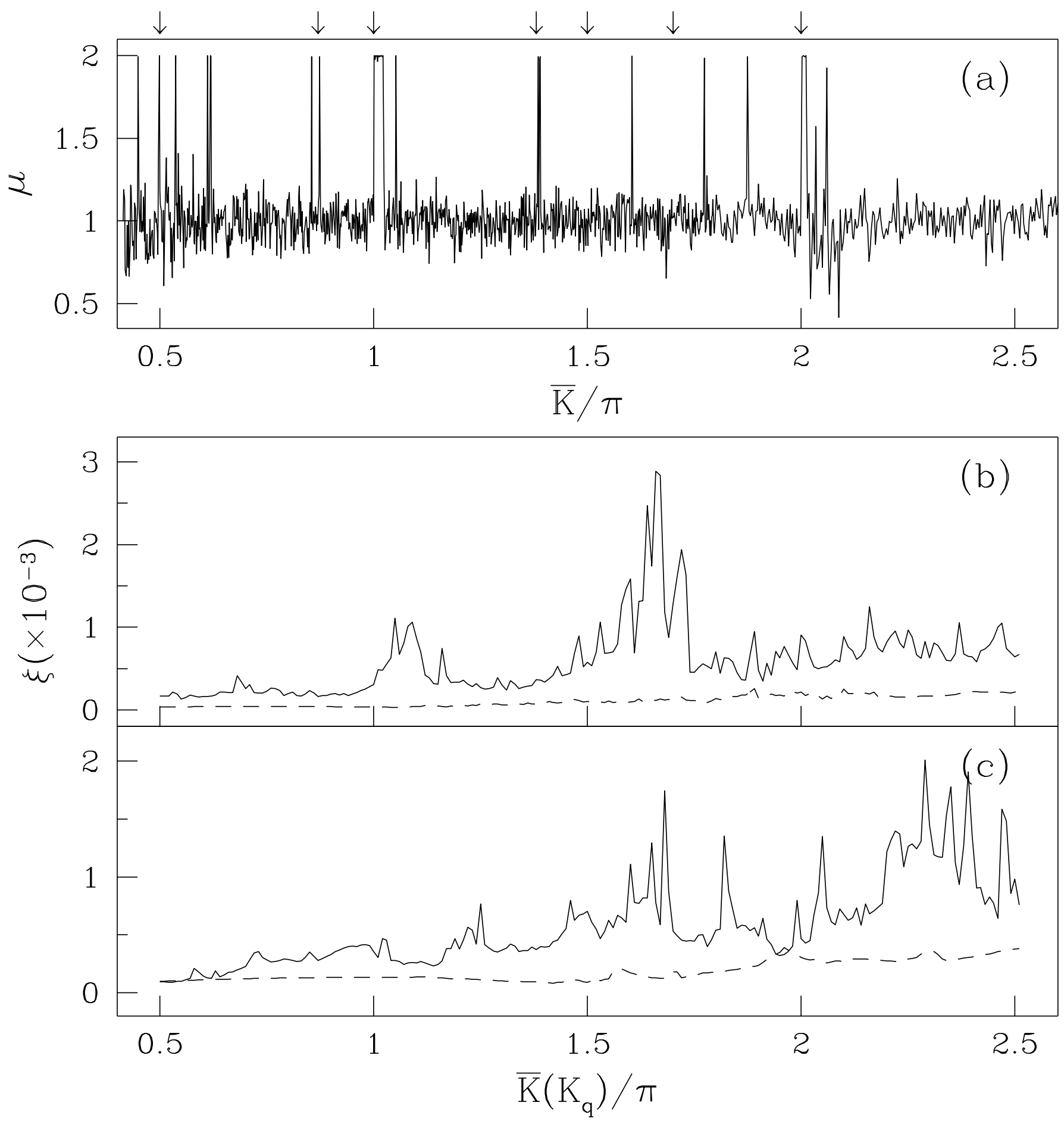


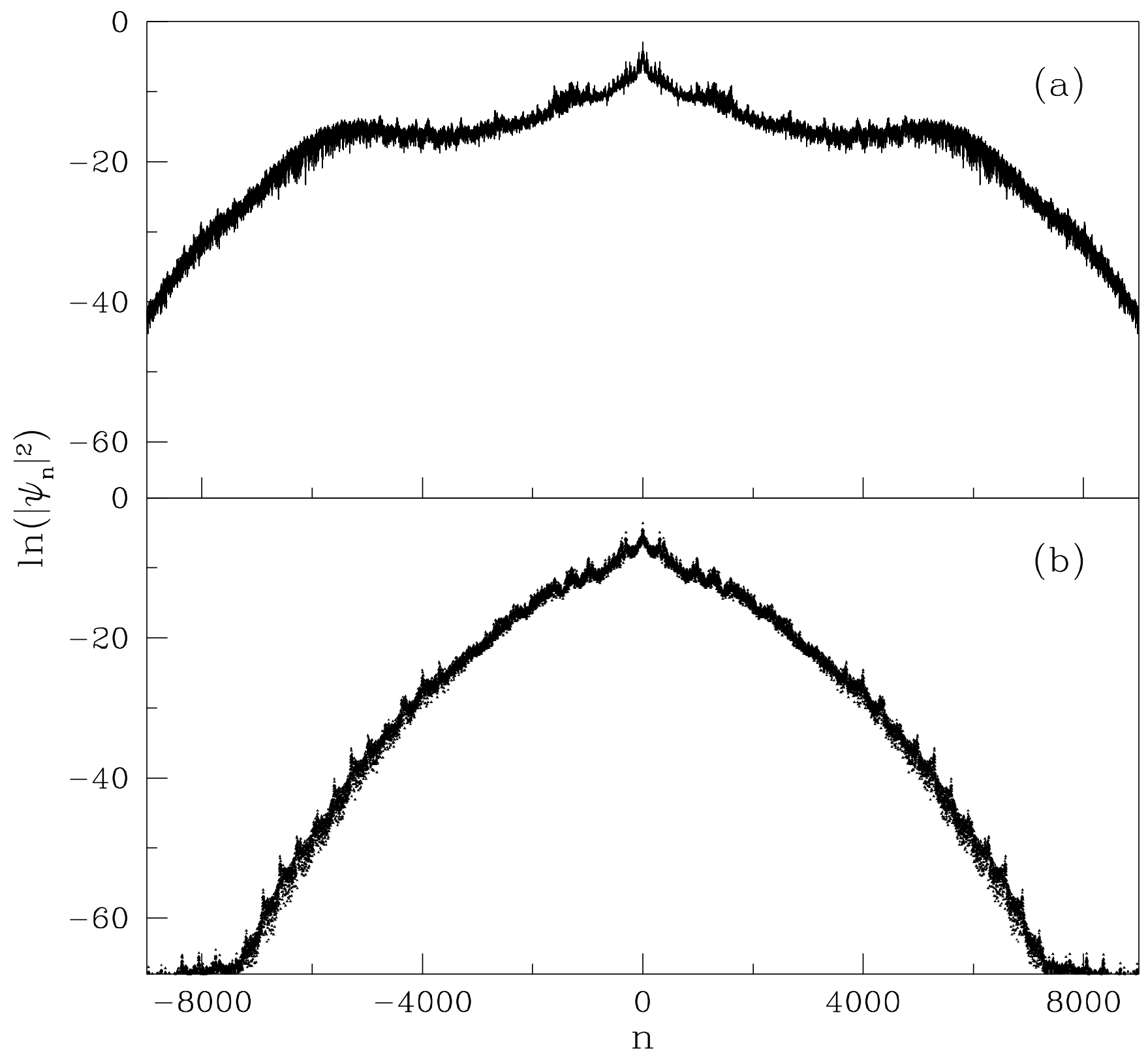

